\title{
METFORMINA: UMA REVISÃO DA LITERATURA
}

\section{Edilson Martins Rodrigues Neto}

Farmacêutico, Doutorando em Farmacologia (Farmacologia Clínica) pela Universidade Federal do Ceará - UFC, Docente dos Cursos de Farmácia, Psicologia e Enfermagem da Faculdade Católica Rainha do Sertão - FCRS, Quixada (CE), Brasil.

E-mail: edilsonmrneto@hotmail.com

\section{Lídia Audrey Rocha Valadas Marques}

Cirurgiã-dentista, Doutoranda em Desenvolvimento e Inovação Tecnológica em Medicamentos programa de pós-graduação em rede das Universidades Federais do Ceará, Rio Grande do Norte, Paraíba e Rural de Pernambuco, Brasil.

\section{Maria Augusta Drago Ferreira}

Farmacêutica; Doutora em Farmacologia pela Universidade Federal do Ceará - UFC, Brasil; Docente Associada do Departamento de Farmácia da Universidade Federal do Ceará - UFC, Brasil.

\section{Patricia Leal Dantas Lobo}

Cirurgiã-dentista, Doutora em Farmacologia pela Universidade Federal do Ceará - UFC, Brasil; Docente Adjunta do Curso de Odontologia da Universidade Federal do Ceará - UFC, Campus Sobral, (CE), Brasil.

\section{Francisco Josimar Girão Junior}

Acadêmico do Curso de Farmácia da Faculdade Católica Rainha do Sertão - FCRS, Quixada (CE), Brasil.

\section{Gisela Costa Camarão}

Farmacêutica, Doutora em Ciências Biológicas (Biologia Molecular) pela Universidade Federal de São Paulo - UNIFESP, Brasil; Docente Associada do Departamento de Fisiologia e Farmacologia da Universidade Federal do Ceará - UFC, Brasil.

\section{Maria Elisabete Amaral de Moraes}

Médica, Doutora em Farmacologia Clínica pela University of Oxford, Reino Unido; Docente Titular de Farmacologia Clínica do Departamento de Fisiologia e Farmacologia da Universidade Federal do Ceará . UFC, Brasil.
RESUMO: A metformina, medicamento comercializado por diversas marcas e também na forma de genérico, é um antidiabético oral pertencente à classe das biguanidas. Devido ao seu perfil de toxicidade e à sua eficácia clínica é a principal escolha no tratamento do diabetes mellitus tipo dois (DM2). Pelo seu grande usoénecessária uma discussão constante a respeito de suas características e aplicações. Objetivou-se realizar uma revisão bibliográfica, na qual serão abordadas a aplicação da metformina no tratamento de DM2 bem como suas propriedades químicas, físico-químicas, farmacocinéticas, farmacodinâmicas, seus usos clínicos, contraindicações e efeitos adversos. Revisão da literatura realizada nos bancos de dados Pubmed, Bireme, Lilacs e Scielo, bem como pesquisa nos periódicos do Portal Brasileiro da Informação Científica e livros sobre o assunto. Pelo exposto percebe-se que a metformina para pacientes diabéticos é considerada um fármaco ideal tendo ainda aplicações na terapêutica adjuvante de outros distúrbios fisiológicos.

PALAVRAS-CHAVE: Biguanidas; Diabetes mellitus tipo 2; Metformina.

\section{METFORMIN: A REVIEW OF THE LITERATURE}

ABSTRACT: Metformin, commercialized under several trademarks and also as a generic medicine, is an oral anti-diabetic drug, belonging to the biguanide class. It is actually the first choice in the treatment of Type 2 diabetes mellitus (DM2) due to its toxicity and clinical efficaciousness. It extensive use requires a constant discussion on its characteristics and applications. Current paper is a bibliographical review comprising the application of metformin in DM2 treatment as its chemical, physical-chemical, pharmacokinetic, pharmacodynamics properties, its clinical usages, contraindications and side effects. Review occurred in Pubmed, Bireme, Lilacs and SciELO data banks and in research in journals published on the Brazilian Platform in Scientific Information and in books on the subject. Results show that metformin is the best medicine for diabetic patients, with auxiliary therapy for other physiological disorders.

KEY WORDS: Biguanides; Diabetes Mellitus type 2; Metformin.

\section{INTRODUÇÃO}

O diabetes é uma síndrome que pode ser definida como uma condição de distúrbios metabólicos heterogêneos caracterizados por hiperglicemia resultante de defeitos na secreção e ação da insulina, ou ambos (ASSOCIAÇÃO AMERICANA DE DIABETES, 2014). 
O diabetes mellitus tipo dois (DM2) é a manifestação majoritária do diabetes, cursando com 90\% dos casos em nível mundial. No ano de 2013 foi declarado, pela Federação Internacional de Diabetes, que 382 milhões de pessoas no mundo convivem com a doença, de forma que $46 \%$ destas não foram diagnosticadas; há uma previsão de 592 milhões de portadores para o ano de 2035 (IDF, 2013). Em 2012 a Sociedade Brasileira de Diabetes anunciou que 12.054.827 indivíduos eram diabéticos no país (SOCIEDADE BRASILEIRA DE DIABETES, 2014).

Essa condição crônica aumenta o risco de desenvolvimento de doenças cardíacas, acidente vascular cerebral e complicações microvasculares. Como manifestações frequentes, pode-se citar a cegueira, falência renal e neuropatia periférica. Atualmente o DM2 é um dos principais agravos de saúde pública em nível global, tendo grande impacto na economia dos governos e dos indivíduos portadores (ASHCROFT; RORSMAN, 2012).

Vários mecanismos estão envolvidos na fisiopatologia da doença, pelo menos oito irregularidades metabólicas ou hormonais podem contribuir para o desenvolvimento da hiperglicemia, entre elas a baixa secreção de insulina, o efeito diminuído da incretina, alta lipólise, reabsorção de glicose nos rins, baixa reabsorção de glicose no músculo esquelético, disfunção de neurotransmissores, produção hepática de glicose aumentada e aumento na secreção de glucagon (DEFRONZO; DAVIDSON; DEL PRATO, 2012).

A metformina, que é um medicamento da classe das biguanidas, é fruto de estudos dos efeitos da Galega officinalis, tendo seu uso relatado desde a Idade Média para o tratamento da poliúria do diabetes, sendo até hoje a principal escolha para o tratamento de DM2. O fármaco apresenta grande eficiência e toxicidade baixa, com poucos efeitos adversos que se delimitam praticamente ao início do tratamento, o que o coloca, no Brasil, na Relação Nacional de Medicamentos Essenciais (BRASIL, 2010a).

Neste trabalho objetivou-se a realizar uma revisão de literatura acerca da metformina, na qual serão abordados diversos aspectos a seu respeito: uso no tratamento de DM2, propriedades químicas e físico- químicas, farmacocinéticas, farmacodinâmicas, seus usos clínicos, contraindicações e efeitos adversos, gerando assim um apanhado universal sobre o fármaco.

\section{MÉTODOS}

Foi realizada uma pesquisa bibliográfica retrospectiva nacional e internacional, utilizando artigos que apresentavam dados e informações relevantes a respeito da metformina (Farmacologia, aplicação clínica e toxicidade). Analisaram-se aspectos gerais sobre o fármaco, sendo as bases de dados eletrônicas utilizadas SCIELO, LILACS e MEDLINE. Os descritores utilizados foram Biguanidas, Diabetes Mellitus tipo 2, Metformina.

\section{REVISÃO DA LITERATURA E DISCUSSÃO}

\subsection{CARACTERÍSTICAS GERAIS DA METFORMINA}

A metformina é o fármaco de primeira escolha para tratamento de DM2, sendo o anti-hiperglicemiante oral mais amplamente prescrito, devido ao seu perfil de toxicidade favorável e eficácia clínica. O fármaco reduz os níveis de glicose principalmente diminuindo a gliconeogênese hepática, o que leva a um declínio médio nos níveis de insulina, e também promove a captação de glicose no músculo (ALEXANDER et al., 2008; BRASIL, 2010).

O cloridrato de metformina é um derivado da guanidina, composto ativo da Galega officinalis. Essa planta medicinal, tendo como sinonímia popular Lilac francês, foi usada por séculos na Europa como tratamento do diabetes desde a época medieval (GRAHAM et al., 2011).

A prática clínica foi complementada por resultados promissores de um estudo realizado no Reino Unido, em que a metformina demonstrou reduzir a incidência de complicações vasculares (infarto do miocárdio, angina, morte súbita, acidente vascular cerebral e doença vascular periférica) em pacientes com excesso de peso e DM2. Este benefício ocorreu de forma independente do controle glicêmico e outros 
fatores de risco cardiovasculares tradicionais (UK PROSPECTIVE DIABETES STUDY, 1998). Contrastando com as sufonilureias e a insulina, a metformina não está associada com risco de hipoglicemia e nem ganho de peso. Além disso, os dados de observação recentes sugerem que, quando comparada com as alternativas anteriormente citadas, a metformina está associada com um risco reduzido de desenvolvimento de câncer de órgão sólido, que depois da doença cardiovascular é a segunda maior causa de morte em pacientes com diabetes (BOLEN; FELDMAN; VASSY, 2007; CURRIE; POLE; GALE, 2009; NYE; HERRINGTON, 2011).

A metformina é o único representante das biguanidas disponível atualmente, sendo um agente insulino-sensibilizador utilizado no tratamento do diabetes mellitus tipo 2 e na síndrome dos ovários policísticos (SOP). É um fármaco com propriedades anti-hiperglicêmicas, que normaliza os níveis elevados de glicose no sangue. Aumenta a sensibilidade à insulina no músculo esquelético, tecido adiposo e, especialmente, no fígado, reduzindo a gliconeogênese hepática e aumentando a captação periférica de glicose (BRANCHTEIN; MATOS, 2004).

$\mathrm{O}$ uso das guanidinas e de seus derivados (fenformina, buformina e metformina) como fármacos para o manejo clínico do DM2 teve início no século passado (GRAHAM et al., 2011).

Há muitos anos, o extrato da planta Galega officinalis demonstrou importante efeito terapêutico ao reduzir os sinais e sintomas clássicos de diabetes mellitus descompensado. Em seguida, quando foi mais bem estudada, demonstrou ser ricaem guanidina, um composto altamente tóxico para o uso clínico corriqueiro. Por isso, em 1920 dois compostos químicos foram sintetizados, decametilene biguanida (Sintalina A) e dodecametilene biguanida (Sintalina B) que demonstraram boa tolerância e eficácia como opção terapêutica para o diabetes. Quase dez anos após, na Alemanha, foi sintetizada a dimetilbiguanida, denominada metformina (GIJS, 2010).

A metformina e a fenformina foram introduzidas na terapêutica em 1957, e a buformina, em 1958. Esta última teve um uso limitado, enquanto a metformina e a fenformina foram amplamente comercializadas. A fenformina foi retirada do mercado em muitos países, durante a década de 1970, devido a uma associação com a manifestação da acidose láctica. A metformina tem sido amplamente prescrita na Europa e no Canadá, e disponível nos EUA desde 1995 (SCHIMMER; PARKER, 2006).

\subsection{CARACTERÍSTICAS QUÍMICAS DA METFORMINA}

A metformina, com fórmula molecular $\mathrm{C}_{4} \mathrm{H}_{11} \mathrm{~N}_{5}$. $\mathrm{HCl}$, é uma dimetilbiguanida, quimicamente, $N, N$ dimetilbiguanida, derivada da guanidina. É um pó branco, cristalino, que é quase inodoro possuindo sabor amargo e propriedades higroscópicas (SIRTORI; PASIK, 1994; BRASIL, 2010b). Seguem, na Tabela 1, dados das características físico-químicas da metformina.

Tabela 1. Características físico-químicas da Metformina

\begin{tabular}{lc}
\hline $\begin{array}{l}\text { Peso molecular na forma de cloridrato de } \\
\text { metformina (base) }\end{array}$ & $165,6(129,2)$ \\
\hline Solubilidade Álcool & $1: 100$ \\
\hline Água & $1: 2$ \\
\hline $\begin{array}{l}\text { Acetona, cloreto de metileno, éter etílico e } \\
\text { clorofórmio }\end{array}$ & $\begin{array}{c}\text { Praticamente } \\
\text { insolúvel }\end{array}$ \\
\hline Faixa de Fusão & 222 a $226^{\circ} \mathrm{C}$ \\
\hline
\end{tabular}

Fonte: Farmacopeia Brasileira (2010).

A metformina é obtida por meio da união de duas moléculas de guanidina e eliminação de amônio. A substituição de dois hidrogênios por grupos metila aumenta a estabilidade metabólica da molécula evitandose a formação de metabólitos potencialmente tóxicos (HOLLENBECK et al., 1991; FILIZOLA; SOUZA JUNIOR; NASCIMENTO, 1995).

\subsubsection{Farmacocinética}

O fármaco cloridrato de metformina é administrado por via oral, absorvido incompleta e lentamente pela parte superior do intestino delgado, tendo sua absorção retardada, mas não prejudicada pela presença de alimentos na bolsa estomacal. No epitélio intestinal, a metformina é absorvida na borda em escova e é um importante substrato da proteína PMAT (plasma 
membrane monoamine transporter). Os transportadores de cátions orgânicos OCT1 e OCT2 estão envolvidos com a entrada da metformina no fígado e rins, respectivamente (ZHOU; XIA; WANG, 2007; TAKANE et al., 2008).

A biodisponibilidade dos comprimidos é da ordem de 50-60\%. A metformina não é metabolizada, circulando em forma livre. A fração ligada a proteínas plasmáticas pode ser considerada como insignificante, sendo distribuída rapidamente para os tecidos periféricos, mas lentamente em eritrócitos. Concentrações mais elevadas do fármaco são encontradas nas glândulas salivares, rins e fígado (NOLTE; KARAN, 2006).

O volume de distribuição aparente do cloridrato de metformina, após doses únicas orais de $850 \mathrm{mg}$ é, em média, de $654 \pm 358$ L. A fração do cloridrato de metformina ligado às proteínas plasmáticas pode ser considerada como insignificante, ao contrário das sulfonilureias, que se ligam em $90 \%$ às proteínas. Em função do tempo, o cloridrato de metformina se compartimentaliza nos eritrócitos. Nas doses clínicas e esquemas de dosagem usuais, as concentrações plasmáticas do cloridrato de metformina no estado de equilíbrio são alcançadas dentro de 24-48 horas e são geralmente $<1 \mu \mathrm{g} / \mathrm{mL}$. Durante estudos clínicos controlados de cloridrato de metformina, os níveis plasmáticos máximos não excederam $5 \mu \mathrm{g} / \mathrm{mL}$, mesmo nas doses máximas (BRASIL, 2010).

A metformina é excretada, majoritariamente, por via urinária de forma inalterada e muito rápida; apesar disso, apresenta uma taxa de biotransformação em torno de $10 \%$, principalmente por reações de fase II, como a metilação ou alquilação dos hidrogênios terminais dos grupamentos amina. Seu clearance, em uma pessoa sadia, é, em média, de $400 \mathrm{~mL} / \mathrm{min}$ (4 a 5 vezes maior que o da creatinina), o que indica filtração glomerular seguida por secreção tubular (BRANCHTEIN; MATOS, 2004).

O fármaco pode acumular-se em nefropatas com depuração da creatinina $<60 \mathrm{~mL} / \mathrm{min}$, o que pode aumentar o risco de acidose láctica. Acumulação também pode ocorrer em idosos devido à função renal reduzida. Estudos de farmacocinética ainda não foram realizados em pacientes com disfunção hepática, apesar de, em teoria, essa condição também poder aumentar o risco de acidose láctica (BRASIL, 2010b). Aspectos farmacocinéticos clinicamente relevantes são descritos na Tabela 2.

Tabela 2. Aspectos farmacocinéticos clinicamente relevantes da metformina

\begin{tabular}{lc}
\hline Absorção & 50 a $60 \%$ \\
\hline Meia-vida & Aproximadamente $6 \mathrm{~h}$ \\
\hline Pico de ação (Cmax) & 1 a $3 \mathrm{~h}$ \\
\hline Efeito máximo & 2 semanas \\
\hline Eliminação & $90 \%$ via renal \\
\hline $\begin{array}{l}\text { Ligação a proteínas } \\
\text { plasmáticas }\end{array}$ & Insignificante \\
\hline
\end{tabular}

Fármaco Dialisável

Fonte: Formulário Terapêutico Nacional (2010).

\subsubsection{Farmacodinâmica}

A redução glicêmica, provocada pela metformina, ocorre principalmente por suas ações no tecido hepático e muscular que apresentam efeito sensibilizador da insulina. No hepatócito, causa a inibição da gliconeogênese e da glicogenólise, assim como a estimulação da glicogênese, enquanto nos tecidos periféricos insulinodependentes, principalmente na musculatura esquelética, aumenta a captação de glicose provocando rápida redução da glicemia. Em contraste com secretagogos, a metformina não aumenta os níveis séricos de insulina e é bem menos passível de causar hipoglicemia, mesmo em doses consideráveis (VIOLLET; FORETZ, 2013).

Somando-se a esses efeitos, ela também diminui a absorção gastrointestinal de glicose, aumenta a sensibilidade à insulina nos tecidos muscular $\mathrm{e}$ adiposo, assim como os níveis de ácidos graxos livres (KIRPICHNIKOV; MCFARLANE; SOWERS, 2002). Nos tecidos periféricos, a metformina intensifica o transporte de glicose ao potencializar a atividade da tirosina quinase nos receptores de insulina. Em nível molecular, a metformina gera muitos efeitos a partir da ativação da proteína quinase ativada por adenosina monofosfato (AMPK) (DOWLING et al., 2011). O mecanismo pelo qual a metformina ativa essa enzima não foi totalmente elucidado; entretanto, demonstrou-se que as biguanidas 
ativam a AMPK indiretamente (OWEN DORAN; HALESTRAP, 2000). A AMPK é uma enzima que induz uma cascata de eventos intracelulares em resposta a mudança da carga elétrica celular (CARLING, 2004; HARDIE, 2003), tendo a AMPK o papel de manutenção da homeostasia energética no metabolismo celular (HARDIE, 2003). Todas as células necessitam continuadamente manter alta relação entre ATP e ADP para realizar suas funções. Esse equilíbrio é obtido por intermédio do catabolismo que aumenta a energia celular convertendo $\mathrm{ADP}$ e fosfato em ATP, enquanto o anabolismo diminui o componente energético celular, por converter ATP em ADP e fosfato. Convém ressaltar o fato de que a relação ATP-ADP nas células geralmente permanece quase constante, indicando a eficiência do mecanismo que regula esse processo, sendo a AMPK um componente chave desse equilíbrio fisiológico (CARLING, 2004; HARDIE, 2003).

Ao ser ativada a AMPK exerce efeitos sobre o metabolismo da glicose e dos lipídios, sobre expressão gênica e sobre síntese proteica. Essa enzima atua em diversos órgãos, incluindo fígado, músculo esquelético, coração, tecido adiposo e pâncreas (ZHOU et al., 2001).

No fígado ocorre a inibição da transcrição das enzimas fosfoenolpiruvato carboxiquinase $\mathrm{e}$ glicose-6-fosfatase, consequentemente reduzindo a gliconeogênese. A AMPK também é responsável pela melhora do metabolismo lipídico durante o tratamento com metformina, pois inibe enzimas chaves na síntese de triglicerídeos e colesterol, respectivamente (ZHOU et al., 2001). No músculo a ativação da AMPK pela metformina promove a utilização de glicose, devido ao aumento da translocação do transportador GLUT4 para a membrana plasmática, e aumento do conteúdo de glicogênio nas células musculares. Além disso, ocorre diminuição da síntese e aumento da oxidação de ácidos graxos (ZHOU et al., 2001).

\subsubsection{Usos Clínicos}

A metformina é um fármaco que apresenta interessante perfil terapêutico, pois além de poder ser utilizado classicamente como agente antidiabético seu emprego se estende a outras situações patológicas, a saber: síndrome metabólica, síndrome do ovário policístico (SOP), hirsutismo e também utilizada como regulador do ciclo menstrual (DOWLING et al., 2011).

Esse fármaco possui a capacidade de alterar o metabolismo lipídico, culminando na redução de triglicérides plasmáticos e ácidos graxos livres, por conta da inibição da lipólise; esse efeito também é associado com diminuição do colesterol total e LDL, assim como aumento discreto do colesterol HDL. A função endotelial também é modulada beneficamente, resultando em uma discreta redução da pressão arterial sistêmica e, além disso, a metformina causa uma redução no peso do paciente por apresentar um efeito anorexígeno e lipolítico. Nesse contexto o fármaco será de grande interesse no manejo da síndrome metabólica, pois tendo em vista todos esses mecanismos percebe-se que há um potencial para reduzir o risco cardiovascular (SANTOMAURO JUNIOR et al., 2008).

A situação patológica de hiperinsulinemia estimula a secreção androgênica ovariana e adrenal, alem de suprimir a produção hepática da globulina transportadora dos hormônios sexuais, resultando no aumento dos androgênios livres biologicamente ativos. O excesso patológico desses androgênios ovarianos pode favorecer a formação de pequenos cistos ovarianos (LEGRO; CASTRACANE; KAUFFMAN, 2004).

A metformina foi empregada inicialmente na abordagem da SOP em um ensaio conduzido por Velasquez, em 1994, visando melhorar a resistência insulínica das pacientes (DUNAIF, 2008). O mecanismo terapêutico da metformina pode ser relacionado à diminuição dos níveis de insulina periférica em pacientes hiperinsulinêmicos e ao aumento do número de receptores carreadores insulínicos (MORIN-PAPUNEN, 2012). O fármaco ainda gera outras repercussões no sistema fisiológico, possuindo uma ação em nível pósreceptor, ao elevar a concentração dos transportadores de glicose tipo 4 nas células responsivas à insulina, o que gera um maior transporte de glicose, por difusão facilitada (VIOLLET; FORETZ, 2013).

O hirsutismo tem como principais opções terapêuticas para seu manejo clínico os anticoncepcionais orais e os antiandrogênios. Em quadros leves, a monoterapia com contraceptivos pode 
ser efetiva (MARTIN et al., 2008). Na impossibilidade de usar o anticoncepcional oral, a metformina, fármaco sensibilizador da ação da insulina, deve ser considerada como uma alternativa para o distúrbio menstrual e tratamento das desordens metabólicas (COSMA et al., 2008).

Em um estudo clínico não-randomizado e nãocontrolado, foram tratadas 15 mulheres não-obesas com SOP, com $2.550 \mathrm{mg}$ /dia de metformina e foi constatada restauração de ciclos ovulatórios e diminuição significativa dos níveis plasmáticos de testosterona (MARCONDES et al., 2007).

\subsubsection{Contraindicações}

O fármaco está contraindicado em casos de: cetoacidose diabética, diabetes gestacional, administração concomitante a anestésicos gerais e contrastes radiográficos, infecções severas, traumatismos e cirurgias, insuficiência renal de qualquer etiologia, hepatopatia, uso abusivo de álcool - possibilidade de dano hepático concomitante e diminuição da oxidação do lactato pelo etanol, enfermidades cardíacas, vasculares ou respiratórias, gestação (BRASIL, 2010a).

\subsubsection{Efeitos Adversos}

Um dos efeitos adversos mais frequentes do uso da metformina é a intolerância gastrointestinal, ocorrendo em torno de $20 \%$ dos pacientes. Cita-se também o gosto metálico, anorexia, náuseas, distensão abdominal e diarreia, os quais, geralmente, são autolimitados e tendem a se resolver com a continuação do tratamento. Uma estratégia para reduzir a ocorrência desses efeitos é a ingestão do medicamento com refeições e a elevação da dose gradual, a cada 7 dias, de acordo com os níveis glicêmicos e até se alcançar as metas terapêuticas. Apenas cerca de 5\% dos pacientes são totalmente intolerantes ao fármaco (MILECH; OLIVEIRA, 2004).

Outro evento adverso associado à metformina é a deficiência de vitamina B12, em tratamentos de longos períodos. Essa correlação vem sendo estudada há algum tempo, porém o mecanismo que leva a esta deficiência ainda não foi totalmente elucidado (TING, 2006).

\section{CONSIDERAÇÕES FINAIS}

Pelo exposto percebe-se que a metformina é um fármaco que apresenta importante papel no tratamento do DM2, tendo ainda aplicações na terapêutica adjuvante de outros distúrbios fisiológicos como a SOP e disfunções androgênicas na mulher.

Além disso, para o paciente diabético, é um fármaco de extrema valia, pois irá contribuir com sua diminuição da massa corporal e na regulação de índices bioquímicos lipídicos.

Sendo assim, a metformina demonstra uma aplicabilidade clínica bastante relevante, também por apresentar perfil de toxicidade e segurança favoráveis e ser bem tolerada durante o tratamento.

\section{REFERÊNCIAS}

ALEXANDER, G. C.; SEHGAL, N. L.; MOLONEY, R. M.; STAFFORD, R. S. National trends in treatment of type 2 diabetes mellitus, 1994-2007. Arch Intern Med, v. 168, p. 2088-2094, 2008.

AMERICAN DIABETES ASSOCIATION - ADA. Diagnosis and classification of diabetes mellitus. Diabetes Care, v. 37, n. 1, p. 81-90, 2014.

ASHCROFT, F. M.; RORSMAN, P. Diabetes Mellitus and the beta cell: the last ten years. Cell, v. 148, n. 1, p. 11601169, 2012.

BOLEN, S.; FELDMAN, L.; VASSY, J. Systematic review: comparative effectiveness and safety of oral medications for type 2 diabetes mellitus. Ann Intern Med., v. 147, n. 1, p. 386-399, 2007.

BRANCHTEIN, L.; MATOS, M. C. G. Farmacologia clínica: fundamentos da terapêutica reacional. 3. ed. Rio de Janeiro: Guanabara Koogan, 2004.

BRASIL. Farmacopeia Brasileira. Agência Nacional de Vigilância Sanitária. Brasília: Anvisa, 2010a. vol. 2, 852p. 
BRASIL. Ministério da Saúde. Secretaria de Ciência, Tecnologia e Insumos Estratégicos. Departamento de Assistência Farmacêutica e Insumos Estratégicos. Formulário terapêutico nacional 2010: Rename 2010. 2. ed. Brasília: Ministério da Saúde, 2010b.

CARLING, D. The AMP-activated protein kinase cascade: a unifying system for energy control. Trends. Biochem. Sci, v. 29 , n. 1 , p. $18-23,2004$.

COSMA, M.; SWIGLO, B. A.; FLYNN, D. N.; KURTZ, D. M.; LABELLA, M. L.; MULLAN, R. J. Clinical review: insulin sensitizers for the treatment of hirsutism: a systematic review and meta analyses of randomized controlled trials.

J. Clin. Endocrinol. Metab., v. 93, n. 4, p. 1135-1142, 2008.

CURRIE, C. J.; POOLE, C. D.; GALE, E. A. The influence of glucose-lowering therapies on cancer risk in type 2 diabetes. Diabetologia, v. 52, n. 1, p. 1766-1777, 2009.

DEFRONZO, R. A.; DAVIDSON, J. A.; DEL PRATO, S. The role of the Kidneys in glucose homeostase: a new path towards normalizing glycemia. Diabetes, Obesity and Metabolism, v. 14, n. 1, p. 5-14, 2012.

DOWLING, R. J. O. et al. Understanding the benefit of metformin use in cancer treatment. BMC Medicine, v. 9, n. 3, 2011.

DUNAIF, A. Drug insight: insulin-sensitizing drugs in the treatment of polycystic ovary syndrome- a reappraisal. Nat Clin Pract Endocrinol. Metab, v. 4, n. 5, p. 272283, 2008.

FILIZOLA, R. G.; SOUZA JUNIOR, E. L.; NASCIMENTO, A. X. Metformina - caracterização bioquímica, mecanismo de ação, indicações e contraindicações na síndrome diabética. Cienc. Cult. Saúde, v. 14, n. 1, p. 14-19, 1995.

GIJS, W. D. Metformin associated with lower cancer mortality in type 2 diabetes. Diabetes Care, v. 33, n. 1, p. 322-326, 2010.

GRAHAM, G. G. et al. Clinical pharmacokinetics of metformin. Clinical pharmacokinetics, v. 50, n. 2, p. 81-98, 2011.

HARDIE, D. G. Minireview: the AMP-activated protein kinase cascade: the key sensor of energy status. Endocrinology, v. 144, n. 1, p. 5179-5183, 2003.

HOLLENBECK, C. B.; JOHSON, P.; VARASTEH, B. B.; CHEN, Y.; REAVEN, G. M. Effects of metformin on glucose, insulin and lipid metabolism in patients with mild hypertriglyceridemia and non-insulin dependent diabetes by glucose tolerance test criteria. Diab. Metabol., v. 17, n. 1, p. 483-489, 1991.

INTERNATIONAL DIABETES FEDERATION. IDF Diabetes Atlas. $6^{\text {th }}$ ed. Brussels, Belgium: International Diabetes Federation, 2013.

KIRPICHNIKOV, D.; MCFARLANE, S.; SOWERS, J. R. Metformin: an update. Ann. Intern. Med., v. 137, n. 1, p. 25-33, 2002 .

LEGRO, R. S.; CASTRACANE, V. D.; KAUFFMAN, R. P. Detecting insulin resistance in polycystic ovary syndrome: purposes and pitfalls. Obstet. Gynecol. Surv., v. 59, n. 2, p. 141-154, 2004.

MARCONDES, J. A.; YAMASHITA, S. A.; MACIEL, G. A.; BARACAT, E. C.; HALPERN, A. Metformin in normalweight hirsute women with polycystic ovary syndrome with normal insulin sensitivity. Ginecol. Endocrinol., v. 23, n. 5, p. 273-278, 2007.

MARTIN, K. A.; CHANG, R. J.; EHRMANN, D. A.; IBANEZ, L.; LOBO, R. A.; ROSENFIELD, R. L. Evaluation and treatment of hirsutism in premenopausal women: an endocrine society clinical practice guideline. J. Clin. Endocrinol. Metab. v. 93, n. 4, p. 1105-1120, 2008.

MILECH, A.; OLIVEIRA, J. E. P. Diabetes mellitus clínica, diagnóstico, tratamento multidisciplinar. São Paulo: Atheneu, 2004.

MORIN-PAPUNEN, L. et al. Metformin improves 
pregnancy and live-birth rates in women with polycystic ovary syndrome (PCOS): a multicenter, double-blind, placebo-controlled randomized trial. The Journal of Clinical Endocrinology \& Metabolism, v. 97, n. 5, p. 1492-1500, 2012.

NOLTE, M. S.; KARAN, J. H. E. Farmacologia básica e clínica. 9. ed. Rio de Janeiro: Guanabara Koogan, 2006.

NYE, H. J.; HERRINGTON, W. G. Metformin: the safest hypoglycaemic agent in chronic kidney disease? Nephron Clinical Practice, v. 118, p. 380-383, 2011.

OWEN, M. R.; DORAN, E.; HALESTRAP, A. P. Evidence that metformin exerts its antidiabetic effects through inhibition of complex 1 of the mitochondrial respiratory chain. Biochem. J., v. 348, n. 1, p. 607-614, 2000.

SANTOMAURO JUNIOR, A. C.; UGOLINI, M. R.; SANTOMAURO, A. T.; SOUTO, R. P. Metformina e AMPK: um antigo fármaco e uma nova enzima no contexto da síndrome metabólica. Arq Bras Endocrinol Metab., v. 52, n. 1, p.120-125, 2008.

SCHIMMER, B. P.; PARKER, K. L. Adrenocorticotropic hormone; adrenocortical steroids and their synthetic analogs: inhibitors of the synthesis and actions of adrenocortical hormones. 11. ed. New York: McGrawHill, 2006.

SIRTORI, C. R.; PASIK, C. Re-evaluation of a biguanide, metformin: mechanism of action and tolerability. Pharmacol Res., v. 30, n. 3, p. 187-228, 1994.

SOCIEDADE BRASILEIRA DE DIABETES. Diretrizes da Sociedade Brasileira de Diabetes: 2013-2014. São Paulo: AC Farmacêutica, 2014.

TAKANE, H.; SHIKATA, E.; OTSUBO, K.; HIGUCHI, S. Polymorphism in human organic cation transporters and metformin action. Pharmacogenomics, v. 9, n. 1, p. 415422, 2008.

TING, R. Z. Risk factors of vitamin B12 deficiency in patients receiveing metformin. Archives Internal Medicine, v. 166, n. 18, p. 1975-1979, 2006.
Effect of intensive blood glucose control with metformin on complications in overweight patients with type 2 diabetes (UKPDS 34). Lancet, v. 352, n. 1, p. 854-856, 1998.

VIOLLET, B.; FORETZ, M. Revisiting the mechanisms of metformin action in the liver. Annales d'endocrinologie, v. 74, n. 2, p. 123-129, 2013.

ZHOU, G.; MYERS, R.; LI, Y.; CHEN, Y.; SHEN, X.; FENYK, M. J. Role of AMP-activated protein kinase in mechanism of metforminaction. J. Clin.Invest., v. 108, n. 1, p. 1167 $1674,2001$.

ZHOU, M.; XIA, L.; WANG, J. Metformin transport by a newly cloned proton-stimulated organic cation transporter (plasma membrane monoamine transporter) expressed in human intestine. Drug Metab. Dispos, v. 35, n. 1, p. 1956-1962, 2007.

Recebido em: 01 de maio de 2015 Aceito em: 14 de junbo de 2015

UK PROSPECTIVE DIABETES STUDY - UKPDS. Group: 